\title{
Involvement of a novel copper chaperone in tyrosinase activity and melanin synthesis in Marinomonas mediterranea
}

\begin{abstract}
Correspondence
A. Sanchez-Amat antonio@um.es
\end{abstract}

Received 9 February 2007

Revised 4 April 2007

Accepted 4 April 2007

\section{López-Serrano, ${ }^{1} \dagger$ F. Solano ${ }^{1}$ and A. Sanchez-Amat ${ }^{2}$}

\author{
${ }^{1}$ Department of Biochemistry and Molecular Biology B, University of Murcia, Murcia 30100, Spain \\ ${ }^{2}$ Department of Genetics and Microbiology, University of Murcia, Murcia 30100, Spain
}

Tyrosinase activity and melanin synthesis in the marine bacterium Marinomonas mediterranea in media with very low copper concentrations are dependent on the presence of a protein (PpoB2) that functions as a chaperone to deliver copper to tyrosinase (PpoB1). Under these conditions, mutants in ppoB2 (such as strain T105) produce PpoB1 as an apoenzyme that can be reconstituted to the active holoenzyme by the addition of cupric ions to cell extracts. To study PpoB2 functionality, a system was developed for genetic complementation in M. mediterranea. Using this approach, melanin synthesis was restored in strain T105 when a wild-type copy of ppoB2 was introduced. $\mathrm{PpoB} 2$ is a novel protein since it is believed to be the first to be described that contains several motifs similar to metal binding motifs present separately in other types of copper-related protein. At least three motifs, a His-rich $\mathrm{N}$-terminal region, and the short $\mathrm{CxxxC}$ and MxxxMM sequences, are essential for the functionality of PpoB2, since site-directed mutagenesis of these motifs resulted in a non-functional protein. In addition, it was demonstrated that PpoB2 is a membrane copper transporter putatively participating in the delivery of this ion specifically to the tyrosinase of $M$. mediterranea and not to a second copper oxidase showing laccase activity that this micro-organism also expresses. PpoB2 has similarities with the COG5486 group encoding putative transmembrane metal binding proteins, and is believed to be the first protein in this group to be experimentally characterized. It may constitute the first example of a novel type of protein involved in copper trafficking in bacteria.

\section{INTRODUCTION}

Copper is an essential micronutrient found in trace amounts in nature (Barceloux, 1999). In cells, it is associated with the active site of numerous enzymes, such as cytochrome $c$ oxidase, tyrosinase, laccase, dopamine $\beta$ hydroxylase, lysyl oxidase and $\mathrm{Cu} / \mathrm{Zn}$ superoxide dismutase (SOD) (Karlin, 1993). However, copper is also known to be highly toxic to cells, due to its tendency to participate in the formation of reactive oxygen species (ROS) such as hydroxyl radicals (Gaetke \& Chow, 2003). Thus, copper ions inside the cell are not free but bound to auxiliary proteins or metallochaperones, which serve as protection against copper toxicity and also shuttle copper ions to the active site of many metalloproteins (Puig \& Thiele, 2002). To control copper homeostasis tightly in cells, copper

tPresent address: Department of Microbiology and Molecular Genetics, Harvard Medical School, 200 Longwood Avenue, Boston, MA 02115, USA.

Abbreviations: DMP, dimethoxyphenol; DMPO, dimethoxyphenol oxidase; DO, DOPA oxidase; DOSDS, DO activated by SDS; $\mathrm{Ha}$, haemagglutinin; PPO, polyphenol oxidase; SOD, superoxide dismutase; $\mathrm{TH}$, tyrosine hydroxylase; $\mathrm{TH}_{\mathrm{SDS}}, \mathrm{TH}$ activated by SDS. membrane transporter (Ctr) proteins have been reported to transport copper from the extracellular medium to the cytoplasm (Zhou \& Thiele, 2001). Copper chaperones are also assumed to protect against copper toxicity by shuttling copper ions to the active site of many metalloproteins. In copper flow from the extracellular space to metalloproteins, copper is coordinated within Ctr proteins with the two methionines of the MxxxM motif, and these proteins mediate the passage of copper ions through the membrane (Puig et al., 2002). In addition, cytoplasmic copper chaperones chelate copper ions, generally by binding with two cysteines from the conserved domain CxxxC, which is crucial for copper chaperone activity (Nittis et al., 2001; Borrelly et al., 2004; Rosenzweig, 2002).

Marinomonas mediterranea is a Gram-negative marine bacterium able to form melanin from L-tyrosine (Solano et al., 1997; Solano \& Sanchez-Amat, 1999). M. mediterranea expresses two copper enzymes with polyphenol oxidase (PPO) activity. The enzyme responsible for melanin synthesis is a tyrosinase, PpoB1, which shows SDSactivated tyrosine hydroxylase $\left(\mathrm{TH}_{\mathrm{SDS}}\right)$ and SDS-activated DOPA oxidase $\left(\mathrm{DO}_{\mathrm{SDS}}\right.$ ) enzymic activities (López-Serrano 
et al., 2002). The second $\mathrm{PPO}$ is PpoA, a multipotent membrane-located laccase not directly involved in melanogenesis (Sanchez-Amat et al., 2001).

Tyrosinase activity is determined by the operon $p p o B$, which consists of two genes, $P$ PoB1, encoding the tyrosinase, and $p p o B 2$, encoding a second protein (LópezSerrano et al., 2004). Mutants in any of these two genes show an amelanogenic phenotype. $M$. mediterranea strain ng56 ( $p p o B 1$ mutant) shows a nonsense point mutation, W122Stop, in the $p p o B 1$ gene. $p p o B 2$ is truncated in $M$. mediterranea strain T105 (ppoB2 mutant) by transposon insertion (López-Serrano et al., 2002). Although both amelanotic mutants are phenotypically identical under standard growth conditions, the addition of extra $\mathrm{CuSO}_{4}$ to the cultures induces the recovery of tyrosinase enzymic activity and melanin synthesis in the $p$ poB2 mutant alone. This effect is not observed in the $p p o B 1$ mutant, which has been described as irreversibly amelanogenic (LópezSerrano et al., 2004). Tyrosinase activity is also recovered in cell extracts from the $p p o B 2$ mutant after copper addition. These results suggest that $\mathrm{PpoB} 2$ is involved in copper transfer to the apotyrosinase encoded by the $p p o B 1$ gene (López-Serrano et al., 2004).

In a similar manner to Marinomonas, most Streptomyces species possess an operon known as melC, which codes for two proteins, MelC2, a tyrosinase, and its copper chaperone, MelC1. MelC1 provides copper to the tyrosinase active site of MelC2 (Lee et al., 1988; Chen et al., 1992). In spite of apparently having similar roles, MelC1 and $\mathrm{PpoB} 2$ show a low overall sequence similarity, except for a His-rich region in the N-terminal fragment of the protein. On the other hand, PpoB2 also shows similarities in other conserved residues to the COG5486 proteins which encode putative metal binding integral membrane proteins (López-Serrano et al., 2004).

So far, no protein similar to PpoB2 has been functionally characterized, and the aim of this study was to determine the cellular localization of PpoB2 and the residues important for its activity. It was observed that it is a membrane protein that delivers copper to the tyrosinase encoded by the $p p o B 1$ gene but not to the second copper PPO, PpoA, expressed by M. mediterranea.

\section{METHODS}

Strains, plasmids, primers and culture media. Bacterial strains, plasmids and primers used in this study are listed in Table 1. Escherichia coli strains were grown in Luria-Bertani medium. $M$. mediterranea was usually grown in Marine Broth, Agar 2216 (Difco) or the complex medium MMC (Fernández et al., 1999). When required, media were supplemented with the appropriate antibiotics (Sigma).

Cellular extraction and enzymic determinations. $M$. mediterranea was grown for $24 \mathrm{~h}$ to stationary phase, when PPO activities are maximal (Lucas-Elío et al., 2002). Cells were harvested by centrifugation at $5000 \mathrm{~g}$ for $6 \mathrm{~min}$. The pellet was washed in SST saline solution (Solano et al., 1997), resuspended in $1 \mathrm{ml} 0.1 \mathrm{M}$ phosphate buffer, $\mathrm{pH} 5.0$, and disrupted by sonication. The homogenate was centrifuged at $14000 \mathrm{~g}$ for $3 \mathrm{~min}$ and the supernatant was used to assay tyrosinase activity by monitoring the oxidation of $2.5 \mathrm{mM}$ L-tyrosine (for TH) or L-DOPA (for $\mathrm{DO})$ at $475 \mathrm{~nm}$ in $0.1 \mathrm{M}$ phosphate buffer, $\mathrm{pH}$ 5.0. For $\mathrm{TH}$ activity, $2.5 \mu \mathrm{M}$ L-DOPA was added to the assay mixture as a cofactor to eliminate any lag period. When required, the activities were also tested in the presence of $0.02 \% \mathrm{SDS}\left(\mathrm{TH}_{\mathrm{SDS}}\right.$ and $\mathrm{DO}_{\mathrm{SDS}}$ ) (Solano et al., 1997). Dimethoxyphenol oxidase (DMPO) activity was determined by monitoring the oxidation of $2 \mathrm{mM}$ dimethoxyphenol (DMP) at $468 \mathrm{~nm}$ in $0.1 \mathrm{M}$ phosphate buffer, $\mathrm{pH}$ 5.0. When copper ions were required in the experiment, $\mathrm{CuSO}_{4}$ was added directly from a $0.1 \mathrm{M}$ stock solution to the cell extract. In all cases, one unit was defined as the amount of enzyme that catalyses the transformation of one micromole of substrate per minute at $37{ }^{\circ} \mathrm{C}$. Specific activities were normalized according to the milligrams of protein present in each sample, measured by bicinchoninic acid assay (Pierce).

Conjugation and transposon mutagenesis. PCR amplification of the $p p o B$ operon from the $p p o B 1$ mutant genome (ng56) was carried out using OPEfw and OPErv primers. To amplify $p p o B 1$ in the wildtype strain (MMB1-R), OPEfw and TYRECOrv primers were used (Table 1). Reverse primers had EcoRI and forward primers had KpnI restriction sites. These sites were used to clone the DNA fragments in a mini-Tn 10 transposon within the pBSL182 vector, creating $\mathrm{pB} p p o B 2$ and pBppoB1. In both cases, PCR amplification included the promoter region.

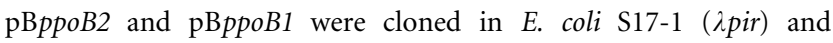
subsequently mobilized into the $p p o B 2$ or $p p o B 1$ mutant by conjugation (Solano et al., 2000). Conjugation plates were enriched with $0.5 \mathrm{mM}$ IPTG, to promote transposition over recombination.

Site-directed mutagenesis. Specific mutations in the $p p o B 2$ gene were generated using forward and reverse pairs of inner primers from the $P p o B 2$ region that was being mutated. Primer pairs were designed with the mutation included in their sequences (HISfw, HISrv; CYSfw, CYSrv; METfw, METrv; Table 1). The ppoB2 gene was amplified in two DNA fragments by PCR using the mutation-carrying inner primers and the outer primers (OPErv and OPEfw). The two DNA fragments obtained were mixed in equal proportions, heated to $95{ }^{\circ} \mathrm{C}$ for $2 \mathrm{~min}$ and placed immediately on ice for $5 \mathrm{~min}$. This mix was used as a template to amplify the entire $p p o B$ operon carrying the mutation. The mutated operons were cloned in pBSL182, checked by sequencing and mobilized to the ppoB2 mutant by conjugation.

Haemagglutinin (Ha) transductional fusion. The Ha epitope DNA sequence was fused to $p p o B 2$ using EcoRI and $B a m H I$ restriction sites. The Ha DNA sequence was obtained by PCR from the pCRE plasmid kindly provided by Professor J. Cansado, University of Murcia. Primers used to amplify the sequence that was cloned into pBppoB2 were TAILfw (EcoRI) and TAILrv (BamHI) (Table 1).

The Ha epitope was detected by Western blotting using a PVDF membrane (Bio-Rad), commercially available antibodies (Sigma), and the ECL Plus kit (Pharmacia-Pfizer).

Bioinformatic tools. Protein sequence alignments were done
according to the CLUSTAL W algorithm with DNASTAR Lasergene
MEGALIGN software (DNASTAR). Protein localization and the
structure of PpoB2 were studied using several bioinformatic tools:
SOSUI (www.expasy.org), provided by the University of Nagoya,
pSORTb (www.psort.org) and ConPred2 (http://bioinfo.si.hirosaki-
u.ac.jp/ ConPred2/). 
Table 1. Strains, plasmids and primers used in this work

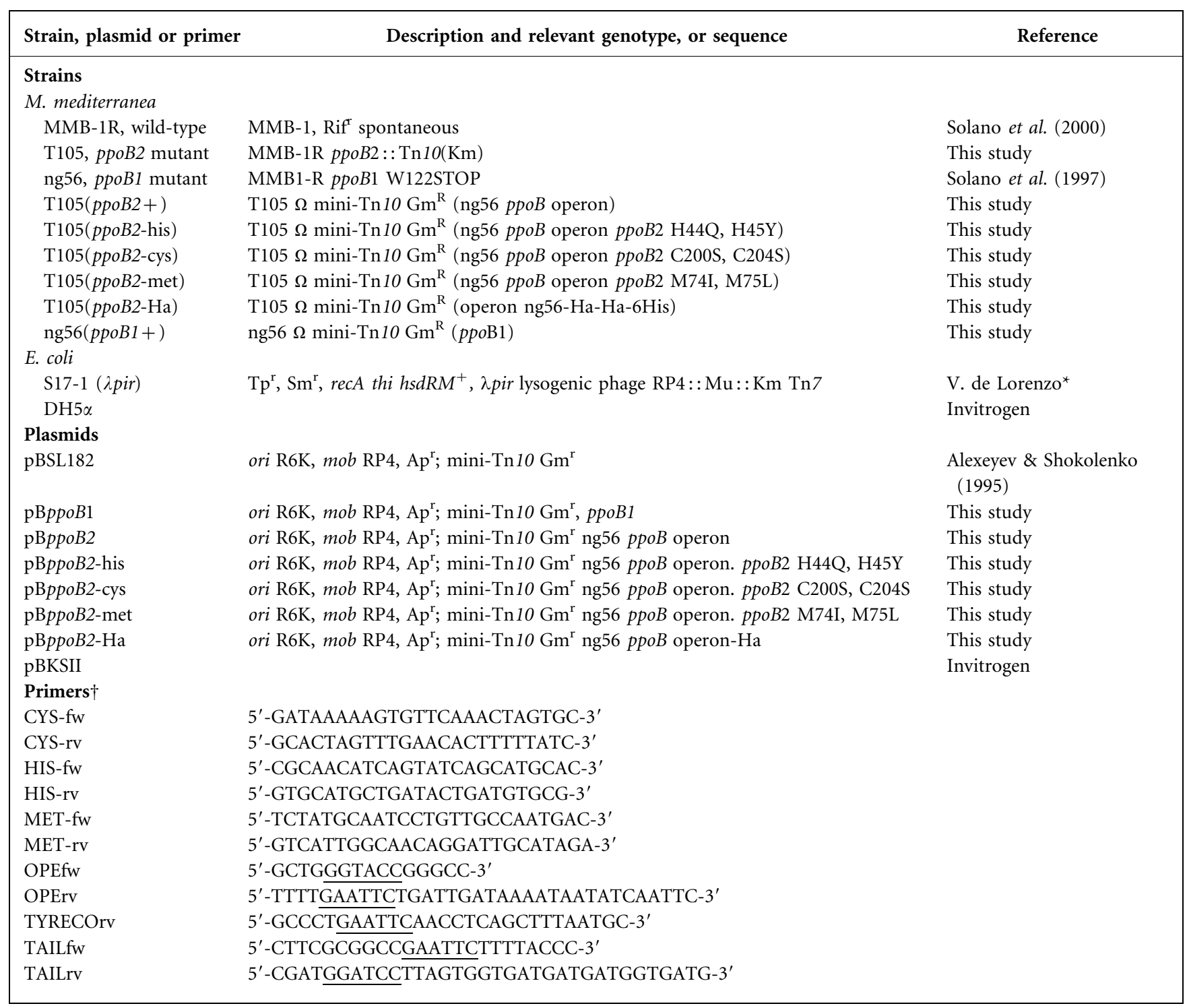

${ }^{*}$ Centro National de Biotecnología, Madrid.

$\dagger$ Restriction sites are underlined.

\section{RESULTS}

\section{PpoB2 mutant characterization by in vitro complementation}

Previous results indicated that $\mathrm{PpoB} 2$ was not required by $M$. mediterranea for tyrosinase activity and melanin synthesis when high copper concentrations, $250 \mu \mathrm{M}$, were added to the culture media. Under these conditions, the wild-type phenotype was partially restored in the ppoB2 mutant, in terms of tyrosinase enzymic activity and melanin synthesis. In addition, the recovery of tyrosinase activity was detected after the direct addition of copper to cell extracts (López-Serrano et al., 2004).
For a more detailed characterization of the effect of copper on the recovery of tyrosinase activity, cell extracts of $p p o B 1$ and ppoB2 mutants (ng56 and T105 strains, respectively) were incubated with a range of $\mathrm{CuSO}_{4}$ concentrations between 0 and $100 \mu \mathrm{M}$ at $25{ }^{\circ} \mathrm{C}$ for $15 \mathrm{~min}$, as tyrosinase copper uptake has been described as a non-instantaneous process (Beltramini \& Lerch, 1982). Tyrosinase activity was not detectable in $p p o B 1$ mutant cell extracts for all copper concentrations assayed (data not shown), consistent with the mutation in the structural gene encoding the tyrosinase. On the other hand, tyrosinase activity in ppoB2 mutant extracts increased when $\mathrm{CuSO}_{4}$ was added (Fig. 1a). The optimum copper concentration to recover maximal $\mathrm{TH}_{\mathrm{SDS}}$ and $\mathrm{DO}_{\mathrm{SDS}}$ enzymic activities of PpoB1 

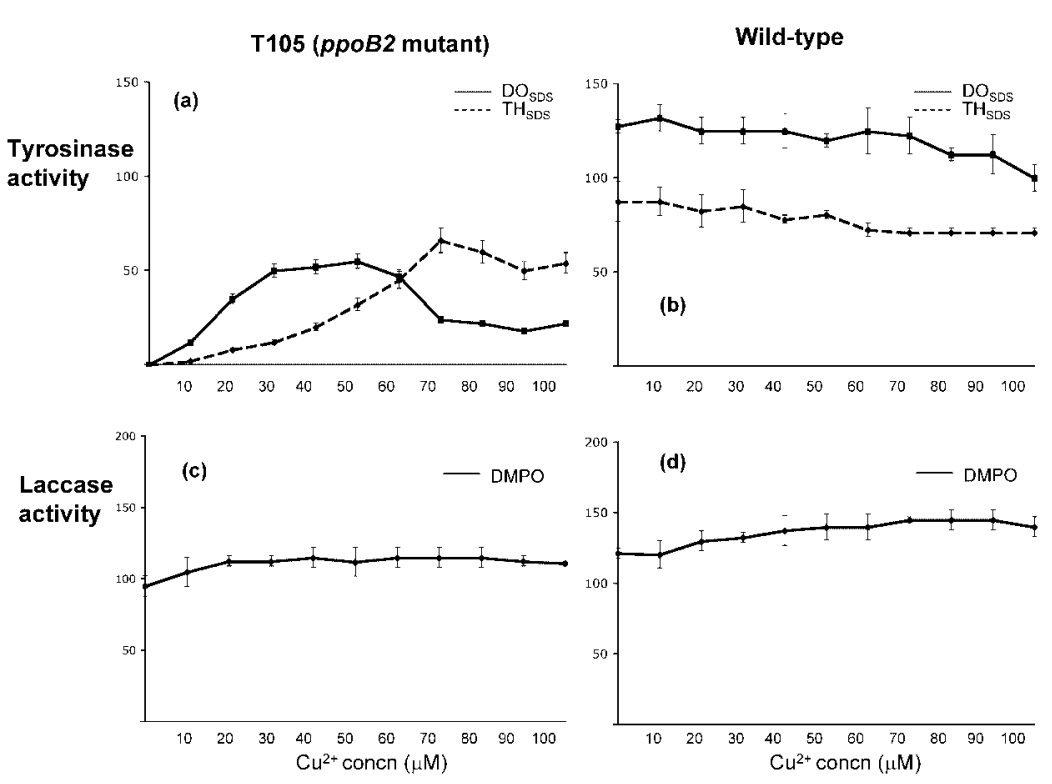

Fig. 1. Measurements of PPO enzymic activities of wild-type and T105, ppoB2 mutant, strains incubated at different copper concentrations. (a, b) $\mathrm{TH}_{\mathrm{SDS}}$ and DO $\mathrm{DDS}$ enzymic activities of extracts of (a) the ppoB2 mutant and (b) the wild-type strain. (c, d) DMPO activity of extracts of (c) the ppoB2 mutant and (d) the wild-type strain. was in the range $50-70 \mu \mathrm{M}$. In contrast, copper addition to the wild-type cell extracts did not lead to an increase in tyrosinase activity, indicating that under standard growth conditions, apotyrosinase was loaded with copper (Fig. 1b).

M. mediterranea expresses two copper PPO enzymes, PpoB1 and PpoA. PpoA shows laccase activity, which is measured using DMP as specific substrate for this enzyme (Solano et al., 2000). Previous results indicated that laccase activity in strain T105 is similar to that of the wild-type strain MMB1-R, suggesting that the absence of PpoB2 does not affect PpoA enzymic activity (López-Serrano et al., 2004). In additional experiments, the response to copper addition of the DMPO activity of cell extracts of wild-type and T105 strains was studied. It was observed that there was no difference between the two strains, and that copper addition did not lead to a significant increase in laccase activity, indicating that the enzyme is copper-loaded in both strains (Fig. 1c, d). Altogether, these results clearly indicate that PpoB2 functionality is not required for copper transfer to the M. mediterranea laccase.

\section{In vivo complementation of the PpoB2 mutation}

In order to study more thoroughly the influence of PpoB2 upon PpoB1 enzymic activities, it was necessary to develop a system for in vivo complementation of the mutations in ppoB2 and ppoB1 mutants. This was achieved for what is believed to be the first time in $M$. mediterranea by using mutagenesis with mini- $\mathrm{Tn} 10$ transposons containing wildtype copies of the genes. These transposons were loaded in conjugative plasmids and mobilized to $M$. mediterranea. Using this protocol, one copy of the ppoB1 wild-type gene along with its promoter was transferred randomly to the genome of the $P p o B 1$ mutant strain ng56 by conjugation, resulting in ng56( $p p o B 1+)$. This mutant contained functional copies of $p p o B 1$ and $p p o B 2$. However, both genes were located in different regions of the genome, and therefore the integrity of the $p p o B$ operon was not restored. The analysis of ng56(ppoB1+) revealed that the strain showed a less intense pigmentation than that of the wildtype strain (Fig. 2a), and in agreement with that, a partial recovery of $\mathrm{TH}_{\mathrm{SDS}}$ and $\mathrm{DO}_{\mathrm{SDS}}$ enzymic activities (Fig. $2 \mathrm{~b}$ ).

The $p p o B$ operon from strain ng56, containing a mutated $p p o B 1$ gene and a wild-type $p p o B 2$ gene, was transferred to strain T105, creating strain T105(ppoB2+). In a similar manner to ng56(ppoB1+), this strain contained functional copies of $p p o B 1$ and $p p o B 2$ located separately in the genome, and also recovered melanin synthesis and tyrosinase activity. However, in contrast to ng56( $p p o B 1+)$, T105( $p$ poB2+) was able to synthesize the same level of melanin (Fig. 3a), and had the same $\mathrm{TH}_{\mathrm{SDS}}$ and $\mathrm{DO}_{\mathrm{SDS}}$ enzymic activities as the wild-type (Fig. $3 \mathrm{~b}$ ).

\section{PpoB2 sequence analysis}

Sequence analysis of PpoB2 showed three putative metal binding motifs, similar to those described in other metalrelated proteins. The motifs were $\mathrm{CxxxC}, \mathrm{MxxxMM}$ and a His-rich $\mathrm{N}$-terminal region. The $\mathrm{CxxxC}$ and MxxxMM motifs are conserved in COG5486 proteins as well as in other hypothetical proteins detected in bacterial genomes (Fig. 4). CxxxC has been described as a copper-acquisition motif in copper-related proteins and copper chaperones (Rosenzweig, 2002; Borrelly et al., 2004). The MxxxMM motif is similar to the MxxxM metal binding motif that appears in membrane copper transporters of yeasts, mediating the passage of copper ions through the membrane (Lee et al., 2002; Puig et al., 2002).

In contrast, the His-rich $\mathrm{N}$-terminal motif of PpoB2 is not present in COG5486 family proteins. However, this motif is similar to other His-rich regions described as copper 
(a)

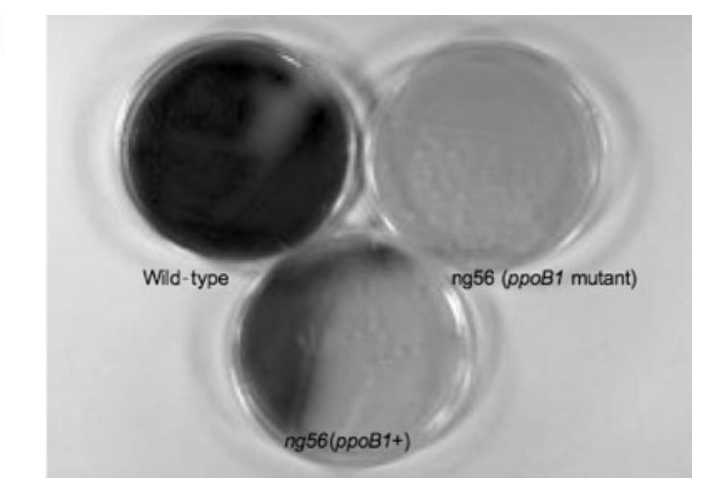

(b)

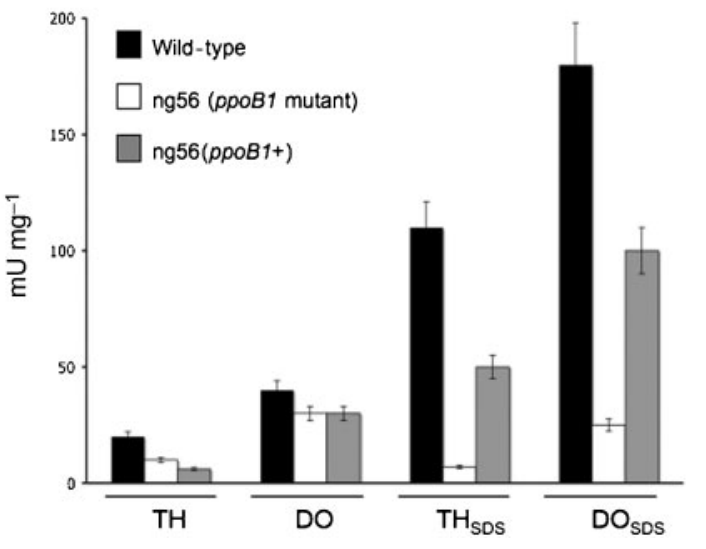

Fig. 2. Comparison of wild-type, ng56 (ppoB1 mutant) and ng56 $(p p o B 1+)$ strains. (a) Melanin synthesis in cultures incubated for 3 days on MMC plates; (b) tyrosinase activities (TH, DO, $\mathrm{TH}_{\mathrm{SDS}}$ and $\left.\mathrm{DO}_{\mathrm{SDS}}\right)$.

binding motifs in, for instance, the MelC1 copper chaperone from Streptomyces (Chen et al., 1993; Liaw \& Lee, 1995) and several bacterial SODs (Battistoni et al., 2001).

To determine how the $\mathrm{CxxxC}, \mathrm{MxxxMM}$ and His-rich Nterminal motifs affect the activity of PpoB2, site-directed mutagenesis was performed on the $p p o B 2$ gene. Cysteines in the CxxxC motif were substituted with serines, which are unable to bind metal ions (C200S; C204S). The two final (a)
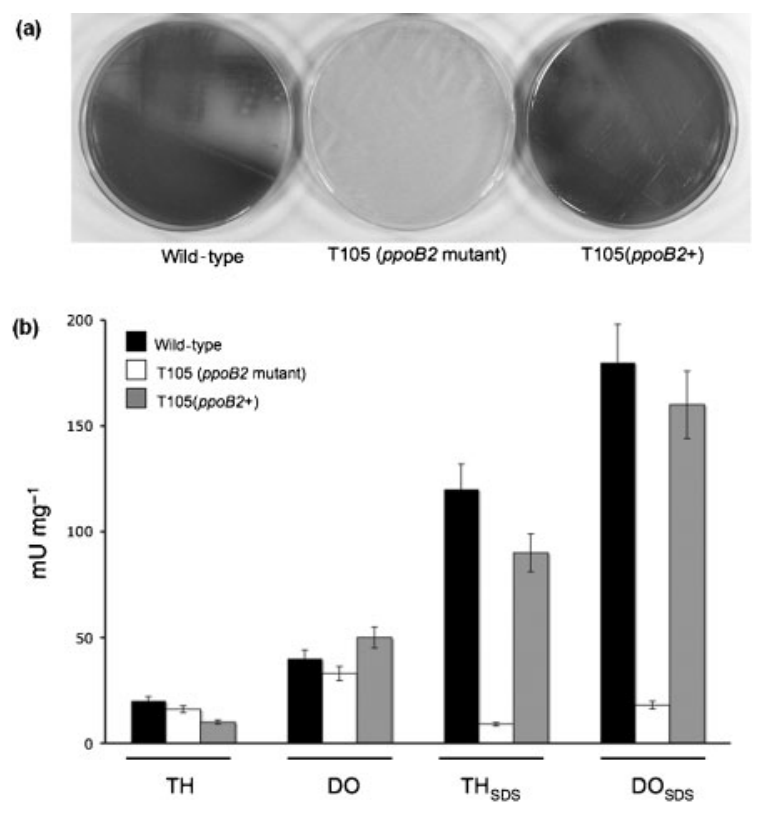

Fig. 3. Comparison of wild-type, T105 ( $p$ poB2 mutant) and T105(ppoB2 +) strains. (a) Melanin synthesis in cultures incubated for 3 days on MMC plates; (b) tyrosinase activities (TH, DO, $\mathrm{TH}_{\mathrm{SDS}}$ and $\left.\mathrm{DO}_{\mathrm{SDS}}\right)$.

methionines in the MxxxMM motif were changed to isoleucine and leucine, respectively (M74I; M75L), to maintain apolarity and steric similarity (Puig et al., 2002). The second and third histidines in the His-rich N-terminal region were substituted with glutamine and tyrosine (H44Q; H45Y), which has some similarity to histidine with respect to size and polarity.

Plasmids with mutated versions of $p$ poB2 were introduced into strain T105 ( $p$ poB2 mutant) by conjugation, using the protocol described above for the wild-type copy of the gene. The characterization of the mutants obtained from these conjugations, strains T105( $p$ poB2-his), T105(ppoB2cys) and T105(ppoB2-met), indicated that all of them showed the same growth rate as strain T105, the only difference being that strain T105( $p$ poB2-his) reached a
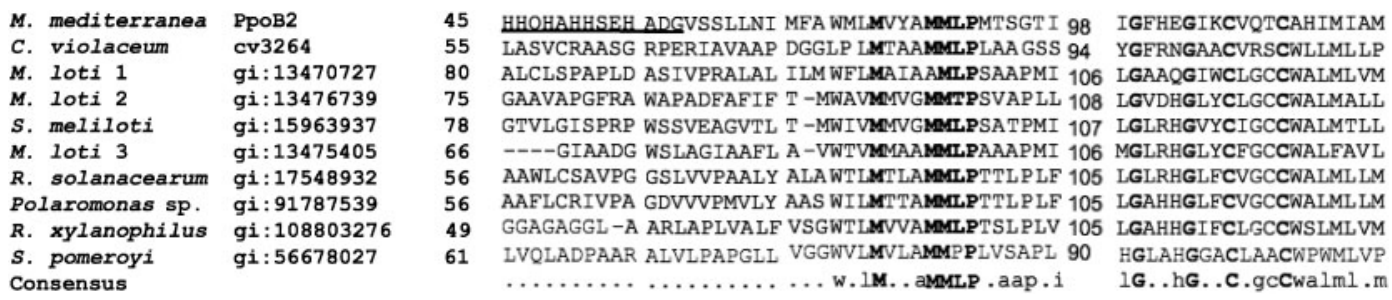

Fig. 4. Alignment of PpoB2 from M. mediterranea with several typical proteins from COG5486. The His-rich region characteristic of PpoB2 is underlined. Conserved residues are marked in bold type and shown in the consensus sequence below. M. loti, Mesorhizobium loti; S. meliloti, Sinorhizobium meliloti; R. xylanophilus, Rubrobacter xylanophilus; S. pomeroyi, Silicibacter pomeroyi. 
slightly lower maximal optical density (data not shown). Strains containing the mutated version of PpoB2 were unable to synthesize melanin (Fig. 5a). Tyrosinase enzymic activities were tested in these three mutants in comparison with the wild-type, T105 and T105(ppoB2+) strains. When no extra copper was added to cell extracts, high levels of $\mathrm{TH}_{\mathrm{SDS}}$ and $\mathrm{DO}_{\mathrm{SDS}}$ activity were detected only in the wildtype and T105(ppoB2+) strains (Fig. 5b), and copper addition to the cell extracts did not increase $\mathrm{TH}_{\mathrm{SDS}}$ or $\mathrm{DO}_{\mathrm{SDS}}$ in these strains. However, strain T105 and its derived strains containing ( $p$ poB2-his), ( $p p o B 2-c y s)$ or (ppoB2-met) showed low tyrosinase activity that increased greatly when extra copper ions were added (Fig. 5c). These phenotypes indicate that PpoB2 with the altered copperrelated motifs is not functional, revealing that the conserved residues detected in this study are essential for its activity.

\section{PpoB2 cellular localization}

Prediction of the topology of PpoB2 was performed by using a range of bioinformatic tools, including SOSUI (Hirokawa et al., 1998), pSORTb (Gardy et al., 2005) and ConPred2 (Arai et al., 2004). All the analyses predicted a membrane localization for PpoB2 with several possible transmembrane regions (Fig. 6a). The topological orientation indicated that the His motif is located in the periplasmic space, the Cys motif in the cytoplasm and the Met motif in the transmembrane region. This prediction is in agreement with the similarity of PpoB2 to the COG5486 group of putative metal-related transmembrane proteins. The cellular localization of PpoB2 was also studied by Western blotting. First, a translational fusion of the Ha epitope to PpoB2 was created, which was then mobilized to strain T105. The new strain, T105( $p$ poB2-Ha), was able to produce melanin and express tyrosinase activity at the same levels as T105(ppoB2+) (data not shown). These results indicate that the Ha fusion did not affect the activity of PpoB2. By Western blotting using anti-Ha antibodies, an extra band of $29 \mathrm{kDa}$ attributable to PpoB2 was detected in the membrane fraction of T105( $p$ poB2-Ha) extracts only (Fig. 6b).

\section{DISCUSSION}

The present study describes the molecular characterization of PpoB2, a novel protein involved in copper delivery to the PpoB1 tyrosinase in M. mediterranea. Strain T105 mutated by transposon insertion in $p p o B 2$ does not show SDS-induced tyrosinase activity, indicating that PpoB1 is not active. The basal levels of TH and DO activity were due to PpoA, an enzyme with laccase activity that also shows $\mathrm{TH}$ and DO activity, though not activated by SDS (Sanchez-Amat \& Solano, 1997). PpoB activity can be recovered by the addition of extra copper to cell cultures or cell extracts. In this study, we have used the second approach to avoid the possible regulatory effect of copper addition on PpoB1 expression. Although this process has not yet been studied in detail in M. mediterranea, it cannot be ruled out that copper might have such an effect, because in several micro-organisms it has been reported that it induces other PPOs, such as laccase (Palmieri et al., 2000).

The results presented in this study clearly indicate that in the absence of PpoB2, PpoB1 is synthesized but remains in the apoprotein form, lacking copper, since the addition of this ion recovers tyrosinase activity. PpoB1 activity can be recovered within an optimum range of copper concentration between 50 and $70 \mu \mathrm{M}$. Furthermore, we noticed that the optimal copper concentrations for $\mathrm{TH}_{\mathrm{SDS}}$ and $\mathrm{DO}_{\mathrm{SDS}}$ enzymic activity were not identical. This effect might be associated with differences in the affinity of the substrate for the PpoB1 active site.

We were also able to determine that the lack of PpoB2 in the ppoB2 mutant does not affect the laccase enzymic (a)

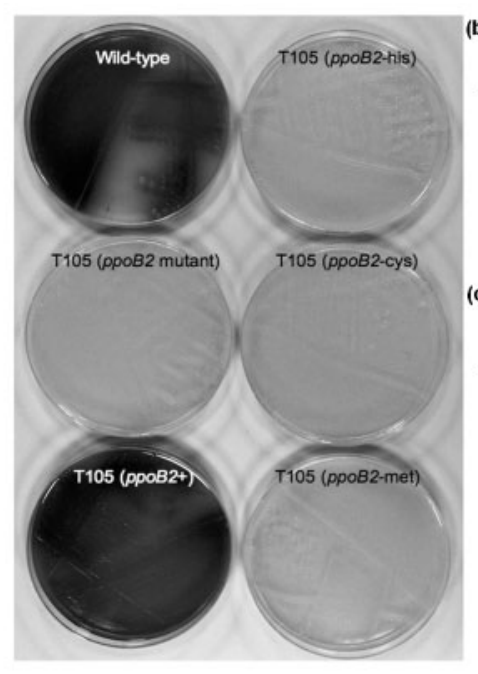

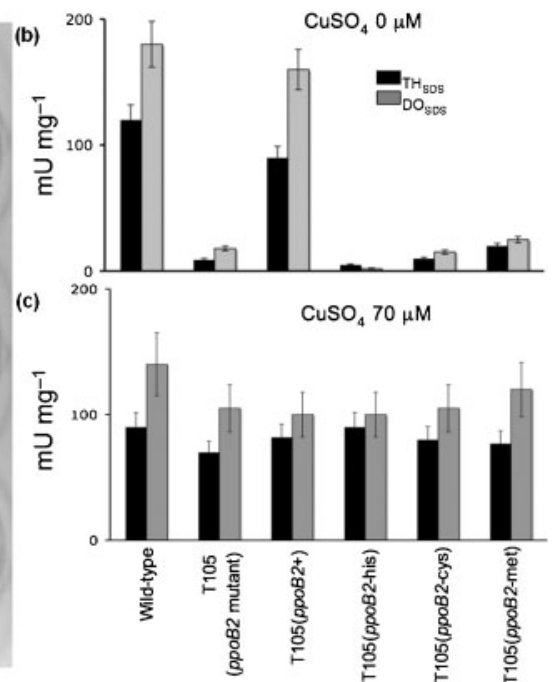

Fig. 5. Comparison of wild-type, T105 (ppoB2 mutant), T105(ppoB2+), T105(ppoB2-his), T105(ppoB2-cys) and T105(ppoB2-met) strains. (a) Melanin synthesis in cultures incubated for 3 days on MMC plates. (b, c) Tyrosinase activities $\left(\mathrm{TH}_{\mathrm{SDS}}\right.$ and $\left.\mathrm{DO}_{\mathrm{SDS}}\right)$ of the mutants when cell extracts were incubated with (b) $0 \mu \mathrm{M}$ and (c) $70 \mu \mathrm{M}$ copper. 
(a)

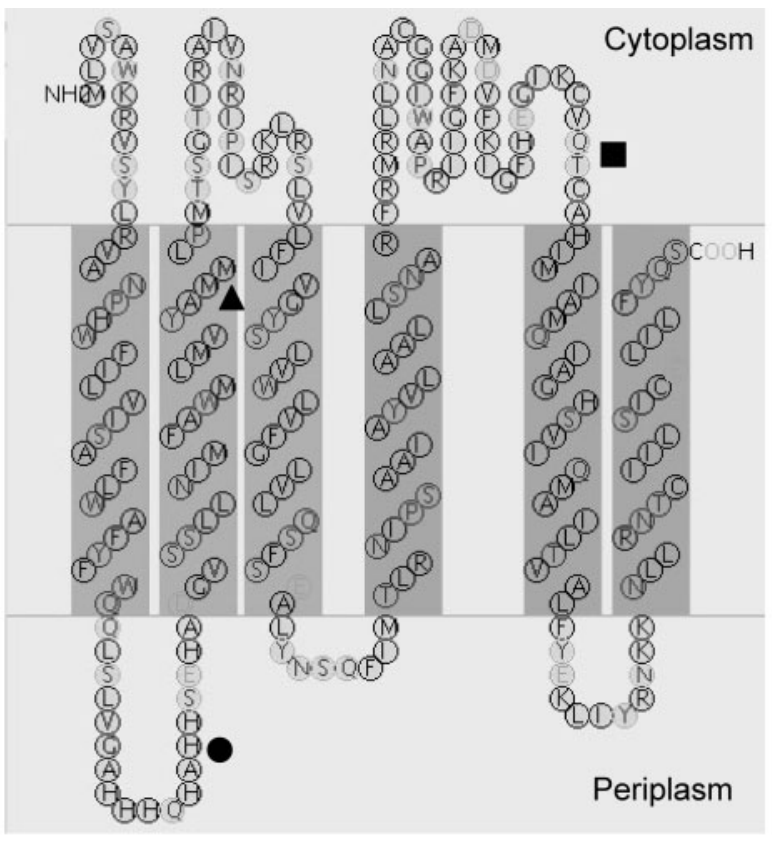

(b)

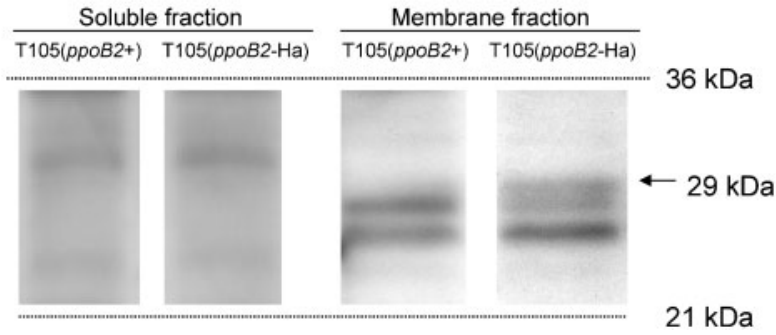

Fig. 6. Membrane localization of PpoB2. (a) Schematic primary structure of PpoB2. Hydrophobic and hypothetical transmembrane regions are represented as grey boxes. Copper binding motifs are marked as $\bullet$ for the His-rich $\mathrm{N}$-terminal region, $\boldsymbol{\Delta}$ for the MxxxMM motif and $\boldsymbol{\square}$ for the CxxxC motif. (b) Western blot of the membrane and cytoplasmic fractions of $p p o B 2$ mutant $(p p o B 2+)$ and $p p o B 2$ mutant $(p p o B 2-\mathrm{Ha})$ mutants using anti-Ha primary antibody.

activity associated with PpoA, clearly indicating that PpoB2 does not shuttle copper to the active site of PpoA, and is therefore exclusive to the PpoB1 tyrosinase. Specific interactions between copper proteins and their copper chaperones have been described in both prokaryotes and eukaryotes. One example is the tyrosinase of Streptomyces, whereby the tyrosinase MelC2 interacts with its copper chaperone MelC1 (Tsai \& Lee, 1998). Another system recently described in Streptomyces is the tyrosinase homologue copper enzyme GriE with $o$-aminophenol oxidase activity, which is encoded in Streptomyces griseus on the same operon as its copper chaperone GriF, which is very similar to MelC1 (Suzuki et al., 2006). In a similar manner to the system in M. mediterranea, mutations in MelC1 lead to an inactive form of MelC2 (Chen et al., 1993). However, MelC1 clearly differs from PpoB2 in several aspects. MelC1 is not a membrane protein, but a small soluble protein that forms a binary complex with the tyrosinase. The formation of this complex is necessary for both copper delivery (Chen et al., 1992) and secretion of tyrosinase (Tsai \& Lee, 1998). The proteins are exported by using the twin-arginine translocation system and a signal peptide present in MelC1 (Schaerlaekens et al., 2001).

Regarding bacterial laccases, so far there are no available data on the mechanisms of copper delivery to these enzymes in bacteria. Our results indicate that $\mathrm{PpoB} 2$ is not involved in copper delivery to PpoA, so a different mechanism must be involved. Sequence analysis in the genomic region surrounding $p p o A$ offers no information, since PpoA does not seem to form part of any operon.

Interaction of PpoB1 and PpoB2 was also shown in vivo by gene complementation experiments. Restoration of melanin synthesis and tyrosinase activity occurs when cells possess at least one copy of each gene, $p p o B 1$ and $p p o B 2$, regardless of whether the integrity of the $p p o B$ operon is restored. However, ng56( $p p o B 1+)$ showed only partial tyrosinase activity and recovery of melanin synthesis. We consider that this could be a consequence of the fact that mutant ng56 ( $p p o B 1-$ ) was obtained by nitrosoguanidine treatment and that other genes involved in the melanogenesis process might have been affected by this treatment. An alternative explanation is that the point mutation in the ppoB1 gene could have a polar effect upon the expression of ppoB2 in strain ng56. We think that this is not the case, since when a transposon containing this operon with the same mutation was introduced into mutant T105 (generated by a transposon insertion affecting $p p o B 2$ alone), a full level of activity and melanogenesis was observed. These results indicate that $p p o B 2$ is expressed in strain ng56 at a normal level, in spite of the upstream mutation in ppoB1. The observation that tyrosinase activity can be restored even when $p p o B 1$ and $p p o B 2$ are in different transcriptional units could be related to the fact that although tyrosinase activity has been described in several Gram-negative bacteria such as Ralstonia solanacearum (HernándezRomero et al., 2005) and Rhizobium (Mercado-Blanco et al., 1993), and that homologues of tyrosinase have been detected by genome sequencing in other strains, the organization of the operon has not been preserved, with the possible exception of Chromobacterium violaceum (López-Serrano et al., 2004). However, genes encoding proteins highly similar to PpoB2 have been detected in the genomes of micro-organisms expressing tyrosinase activity, suggesting that the requirement for a copper chaperone is common in bacterial tyrosinases.

PpoB2 is believed to be the first described case of a copperrelated protein which presents several motifs similar to metal binding motifs described in diverse copper proteins. These are the CxxxC, MxxxMM and His-rich N-terminal motifs. Site-directed mutagenesis of the three copper binding motifs resulted in three different versions of ppoB2, none of which was able to complement the mutation in strain T105 and recover the ability to form 
melanin, in contrast to the wild-type phenotype observed in $\mathrm{T} 105(p p o B 2+)$.

Excluding the His-rich $\mathrm{N}$-terminal region, in the other two motifs, PpoB2 shows high similarity to the COG5486 group of putative metal binding transmembrane proteins. Our results confirmed that PpoB2 is indeed a membrane protein. Several of the motifs have been studied in membrane proteins. Cysteine motifs are present in several types of copper chaperone (Harrison et al., 2000). In particular, the CxxxC metal binding motif is conserved in all Scol homologue copper chaperones (Swem et al., 2005). Scol is localized in the inner mitochondrial membrane, and the CxxxC motif is involved in copper transfer to the cytochrome oxidase COX. MxxxMM is similar to the MxxxM motif present in membrane copper transporters in yeasts; in those proteins it is located in the transmembrane region, and it is postulated that it works in copper passage through the membrane (Puig et al., 2002).

Regarding the His-rich motif in the N-terminal region, a similar region has been reported in some metalloproteins such as the Cu/Zn SOD from Haemophilus ducreyi and Haemophilus parainfluenzae. In these cases, it has been postulated that this region is involved in metal binding by the enzyme under conditions of limited copper availability. Later on, copper is transferred from the His-rich region to the active centre of the enzyme (Battistoni et al., 2001).

The results presented in this study point to a model of PpoB2 activity in which this membrane protein is involved in the transport and delivery of copper to the tyrosinase when the concentration of this ion is low in the external medium. At high copper concentrations it might act an alternative mechanism, explaining why mutants in PpoB2 show normal levels of tyrosinase activity and melanin synthesis under these conditions (López-Serrano et al., 2004). Tyrosinase activity has been detected in the soluble fraction of M. mediterranea culture extracts (Fernández et al., 1999). The membrane localization of PpoB2 suggests that it is involved in copper trafficking between different compartments. The confirmation of this hypothesis would require further studies to determine the topological orientation of PpoB2 fragments, but an interesting possibility suggested by our results is that the His-rich region binds copper in the periplasmic space. The copper may pass through the membrane by the transmembrane fragments containing the conserved Met residues, and finally the Cys motif would be involved in direct copper delivery to the tyrosinase.

Interestingly, estuaries, reefs and coastal sea waters in the Mediterranean sea are described as poor in copper (0.4$1.8 \mu \mathrm{g} \mathrm{l}^{-1}$ ), compared to the higher copper concentrations in ocean seawaters $\left(0.83-8.10 \mu \mathrm{g} \mathrm{l}^{-1}\right)$ (van Geen, 1989). Accordingly, marine bacteria have developed a variety of means to acquire this and other transition metals (Butler, 1998). The expression of PpoB2 in the marine bacterium $M$. mediterranea can facilitate copper transfer to the tyrosinase and ensure the functionality of this enzyme in copper-depleted marine waters.
PpoB2 is a novel example of a protein involved in copper delivery to a metalloprotein. The elucidation of its mechanism of action would increase the knowledge of this important biological process. Proteins similar to PpoB2 have been detected by sequencing of the genomes of different organisms. It would be interesting to determine whether these PpoB2-like proteins are involved in copper transfer to tyrosinases or whether, alternatively, in other micro-organisms they participate in the delivery of copper or other metals to different enzymes.

\section{ACKNOWLEDGEMENTS}

This work was supported by grant PB2004-4803 from the Ministry of Education and Science (MEC), Spain. D. L.-S. was a recipient of a pre-doctoral fellowship from the University of Murcia. Thanks to N. Skinner (Universidad Autonoma de Barcelona) and all the members of the Kolter Laboratory (Harvard Medical School) for their helpful comments and discussions.

\section{REFERENCES}

Alexeyev, M. F. \& Shokolenko, I. N. (1995). Mini-Tn 10 transposon derivatives for insertion mutagenesis and gene delivery into the chromosome of Gram-negative bacteria. Gene 160, 59-62.

Arai, M., Mitsuke, H., Ikeda, M., Xia, J.-X., Kikuchi, T., Satake, M. \& Shimizu, T. (2004). ConPred II: a consensus prediction method for obtaining transmembrane topology models with high reliability. Nucleic Acids Res 32, W390-W393.

Barceloux, D. G. (1999). Copper. J Toxicol Clin Toxicol 37, 217-230.

Battistoni, A., Pacello, F., Mazzetti, A. P., Capo, C., Kroll, J. S., Langford, P. R., Sansone, A., Donnarumma, G., Valenti, P. \& Rotilio, G. (2001). A histidine-rich metal binding domain at the $\mathrm{N}$ terminus of $\mathrm{Cu}, \mathrm{Zn}$-superoxide dismutases from pathogenic bacteria: a novel strategy for metal chaperoning. J Biol Chem 276, 30315-30325.

Beltramini, M. \& Lerch, K. (1982). Copper transfer between Neurospora copper metallothionein and type 3 copper apoproteins. FEBS Lett 142, 219-222.

Borrelly, G. P., Blindauer, C. A., Schmid, R., Butler, C. S., Cooper, C. E., Harvey, I., Sadler, P. J. \& Robinson, N. J. (2004). A novel copper site in a cyanobacterial metallochaperone. Biochem J 378, 293-297.

Butler, A. (1998). Acquisition and utilization of transition metal ions by marine organisms. Science 281, 207-210.

Chen, L.-Y., Leu, W.-M., Wang, K.-T. \& Wu Lee, Y.-H. (1992). Copper transfer and activation of the Streptomyces apotyrosinase are mediated through a complex formation between apotyrosinase and its transactivator MelC1. J Biol Chem 267, 20100-20107.

Chen, L. Y., Chen, M. Y., Leu, W. M., Tsai, T. Y. \& Lee, Y. H. (1993). Mutational study of Streptomyces tyrosinase trans-activator MelC1. MelC1 is likely a chaperone for apotyrosinase. J Biol Chem 268, 18710-18716.

Fernández, E., Sanchez-Amat, A. \& Solano, F. (1999). Location and catalytic characteristics of a multipotent bacterial polyphenol oxidase. Pigment Cell Res 12, 331-339.

Gaetke, L. M. \& Chow, C. K. (2003). Copper toxicity, oxidative stress, and antioxidant nutrients. Toxicology 189, 147-163.

Gardy, J. L., Laird, M. R., Chen, F., Rey, S., Walsh, C. J., Ester, M. \& Brinkman, F. S. L. (2005). PSORTb v.2.0: expanded prediction of bacterial protein subcellular localization and insights gained from comparative proteome analysis. Bioinformatics 21, 617-623. 
Harrison, M. D., Jones, C. E., Solioz, M. \& Dameron, C. T. (2000). Intracellular copper routing: the role of copper chaperones. Trends Biochem Sci 25, 29-32.

Hernández-Romero, D., Solano, F. \& Sanchez-Amat, A. (2005). Polyphenol oxidase activity expression in Ralstonia solanacearum. Appl Environ Microbiol 71, 6808-6815.

Hirokawa, T., Boon-Chieng, S. \& Mitaku, S. (1998). SOSUI: classification and secondary structure prediction system for membrane proteins. Bioinformatics 14, 378-379.

Karlin, K. D. (1993). Metalloenzymes, structural motifs, and inorganic models. Science 261, 701-708.

Lee, Y. H., Chen, B. F., Wu, S. Y., Leu, W. M., Lin, J. J., Chen, C. W. \& Lo, S. C. (1988). A trans-acting gene is required for the phenotypic expression of a tyrosinase gene in Streptomyces. Gene 65, 71-81.

Lee, J., Pena, M. M., Nose, Y. \& Thiele, D. J. (2002). Biochemical characterization of the human copper transporter Ctr1.J Biol Chem 277, 4380-4387.

Liaw, L. L. \& Lee, Y. H. (1995). Histidine residues 102 and 117 of $\mathrm{MelC1}$ play different roles in the chaperone function for Streptomyces apotyrosinase. Biochem Biophys Res Commun 214, 447-453.

López-Serrano, D., Sanchez-Amat, A. \& Solano, F. (2002). Cloning and molecular characterization of a SDS-activated tyrosinase from Marinomonas mediterranea. Pigment Cell Res 15, 104-111.

López-Serrano, D., Solano, F. \& Sanchez-Amat, A. (2004). Identification of an operon involved in tyrosinase activity and melanin synthesis in Marinomonas mediterranea. Gene 342, 179-187.

Lucas-Elío, P., Solano, F. \& Sanchez-Amat, A. (2002). Regulation of polyphenol oxidase activities and melanin synthesis in Marinomonas mediterranea: Identification of $p p o S$, a gene encoding a sensor histidine kinase. Microbiology 148, 2457-2466.

Mercado-Blanco, J., Garcia, F., Fernandez-Lopez, M. \& Olivares, J. (1993). Melanin production by Rhizobium meliloti GR4 is linked to nonsymbiotic plasmid pRmeGR4b: cloning, sequencing, and expression of the tyrosinase gene mepA. J Bacteriol 175, 5403-5410.

Nittis, T., George, G. N. \& Winge, D. R. (2001). Yeast Sco1, a protein essential for cytochrome $c$ oxidase function is a $\mathrm{Cu}(\mathrm{I})$-binding protein. J Biol Chem 276, 42520-42526.

Palmieri, G., Giardina, G., Bianco, C., Fontanella, B. \& Sannita, G. (2000). Copper induction of laccase isoenzymes in the ligninolytic fungus Pleurotus ostreatus. Appl Environ Microbiol 66, 920-924.

Puig, S. \& Thiele, D. J. (2002). Molecular mechanisms of copper uptake and distribution. Curr Opin Chem Biol 6, 171-180.

Puig, S., Lee, J., Lau, M. \& Thiele, D. J. (2002). Biochemical and genetic analyses of yeast and human high affinity copper transporters suggest a conserved mechanism for copper uptake. J Biol Chem 277, 26021-26030.

Rosenzweig, A. C. (2002). Metallochaperones: bind and deliver. Chem Biol 9, 673-677.

Sanchez-Amat, A. \& Solano, F. (1997). A pluripotent polyphenol oxidase from the melanogenic marine Alteromonas sp shares catalytic capabilities of tyrosinases and laccases. Biochem Biophys Res Commun 240, 787-792.

Sanchez-Amat, A., Lucas-Elio, P., Fernández, E., Garcia-Borrón, J. C. \& Solano, F. (2001). Molecular cloning and functional characterization of a unique multipotent polyphenol oxidase from Marinomonas mediterranea. Biochim Biophys Acta 1547, 104-116.

Schaerlaekens, K., Schierova, M., Lammertyn, E., Geukens, N., Anne, J. \& Van Mellaert, L. (2001). Twin-arginine translocation pathway in Streptomyces lividans. J Bacteriol 183, 6727-6732.

Solano, F. \& Sanchez-Amat, A. (1999). Studies on the phylogenetic relationships of melanogenic marine bacteria: proposal of Marinomonas mediterranea sp. nov. Int J Syst Bacteriol 49, 1241-1246.

Solano, F., García, E., Pérez de Egea, E. \& Sanchez-Amat, A. (1997). Isolation and characterization of strain MMB-1 (CECT 4803), a novel melanogenic marine bacterium. Appl Environ Microbiol 63, 3499-3506.

Solano, F., Lucas-Elío, P., Fernández, E. \& Sanchez-Amat, A. (2000). Marinomonas mediterranea MMB-1 transposon mutagenesis: isolation of a multipotent polyphenol oxidase mutant. J Bacteriol 182, 3754-3760.

Suzuki, H., Furusho, Y., Higashi, T., Ohnishi, Y. \& Horinouchi, S. (2006). A novel $o$-aminophenol oxidase responsible for formation of the phenoxazinone chromophore of grixazone. J Biol Chem 281, 824-833.

Swem, D. L., Swem, L. R., Setterdhal, A. \& Bauer, C. E. (2005). Involvement of SenC in assembly of cytochrome $c$ oxidase in Rhodobacter capsulatus. J Bacteriol 187, 8081-8087.

Tsai, T.-Y. \& Lee, Y.-H. (1998). Roles of copper ligands in the activation and secretion of Streptomyces tyrosinase. J Biol Chem 273, 19243-19250.

van Geen, A. F. M. J. (1989). Trace metal sources for the Atlantic inflow to the Mediterranean Sea. $\mathrm{PhD}$ thesis, Massachusetts Institute of Technology. Bibliographic code: 1989PhDT-66V.

Zhou, H. \& Thiele, D. J. (2001). Identification of a novel high affinity copper transport complex in the fission yeast Schizosaccharomyces pombe. J Biol Chem 276, 20529-20535.

Edited by: T. J. Donohue 